\title{
CARATTERISTICHE MINERALOGICHE DELLE GROTTE DEL CARSO TRIESTINO
}

\author{
Graziano Cancian * e Francesco Princivalle *
}

\section{INTRODUZIONE}

Andando nelle grotte ci si sofferma soprattutto ad ammirare la bellezza delle concrezioni calcaree, in qualunque forma esse si manifestino. Le incrostazioni, presenti sia sulle volte, sia sulle pareti, o peggio ancora le fanghiglie del suolo, molto spesso vengono trascurate, ma proprio queste mostrano una gran varietà di minerali e talvolta anche delle rarità, dovute alle particolari condizioni della grotta in cui si sono formate. Da qualche tempo ci si occupa anche di queste "cenerentole" delle grotte, aiutati dagli speleologi che sembrano ammaliati da tutto ciò che non ha l'aspetto della calcite.

In questa breve esposizione trascureremo pertanto la calcite, di cui già tanto si è scritto, e ci occuperemo invece degli altri minerali. Inoltre, poiché lo studio sistematico della mineralogia delle grotte è appena agli inizi, gli argomenti trattati non saranno ovviamente esaurienti, dato che necessitano di ulteriori studi.

\section{LA TERRA ROSSA}

Il suolo delle caverne del Carso spesso è caratterizzato dalla presenza di "terre rosse" generalmente fluitate. Su queste sono state eseguite delle analisi diffrattometriche, che hanno messo in evidenza i seguenti minerali: quarzo, feldspati e argille (prevalentemente illite, seguita da clorite, caolinite e smectite). La calcite è presente in quantità variabili, anche se talvolta risulta assente. Il confronto con le terre rosse presenti all'esterno delle grotte, ha evidenziato come in queste ultime la calcite sia generalmente più scarsa (CANCIAN, CHIORBOLI, LENZI, 1986).

* Società di studi carsici “A.F. Lindner” - Fogliano (GO).

* * Istituto di Mineralogia e Petrografia - Trieste. 


\section{I DEPOSITI ALLUVIONALI}

In alcune grotte e relitti di cavità del Carso goriziano sono stati trovati dei depositi di sabbie e ghiaie fini, in quantità variabili da pochi granuli fino a diversi metri cubi. A questo proposito è interessante segnalare che il gruppo Speleologico "Talpe del Carso", ha recentemente scoperto un discreto deposito di sabbie e ghiaie fini, essenzialmente quarzose, in un nuovo ramo della Grotta del Monte San Michele (450 VG). L'origine di tale deposito, proprio per la presenza dei ciottolini quarzosi risulta dubbia e lascia aperto il campo a numerose ipotesi. Sono inoltre presenti, anche se in minori quantità, dei granuli neri, composti da quarzo, diasporo [AlO $(\mathrm{OH})$ fase beta], bohemite $[\mathrm{AlO}(\mathrm{OH})$ fase gamma], goethite $[\mathrm{FeO}(\mathrm{OH})]$, la cui composizione è assimilabile alle bauxiti ferrose.

Depositi analoghi sono più frequenti in sacche e relitti di cavità del Carso monfalconese (ANDREOLOTTI, 1966, 1970; CANCIAN, 1981).

Nei riempimenti di diverse caverne della provincia di Trieste compare un livello di "sabbie siltose gialle" (livello $\mathrm{B}_{2}$ in ANDREOLOTTI, 1965; FORTI, 1973), composto da quarzo e subordinatamente argille e feldspati. Questo livello può derivare sia dal disfacimento di formazioni di Flysch, sia da antiche alluvioni, sia da trasporto eolico.

\section{I FOSFATI}

In molte cavità naturali del Carso vivono - o vivevano nel passato numerose colonie di pipistrelli, che hanno dato origine a cumuli di guano. Dalla decomposizione di questo materiale organico si sono originate soluzioni ricche di nitrati e di fosfati, le quali reagendo con le soluzioni carbonatiche e con gli stessi carbonati, hanno portato alla formazione di idrossiapatite - Cas $\left(\mathrm{PO}_{4}\right)_{3}(\mathrm{OH})-\mathrm{o}$ di brushite $-\mathrm{CaHPO}_{4}, 2 \mathrm{H}_{2} \mathrm{O}$.

Nel suolo questi due minerali hanno l'aspetto di masserelle irregolari, tenere e di colore biancastro. Solo in alcuni casi sono stati rinvenuti dei noduli di brushite duri e di colore marroncino (abisso di Fernetti 88 VG; CANCIAN, 1987).

Nelle volte e sulle pareti di numerose grotte del Carso goriziano sono state rinvenute incrostazioni fosfatiche generalmente dure e di colore marroncino o grigio (CANCIAN et al., 1990). Queste incrostazioni sono rappresentate quasi esclusivamente da idrossiapatite, mentre la brushite è presente solo in rari casi. Ciò può essere relazionato al $\mathrm{PH}$ delle acque di percolazione, generalmente superiore a 7 . L'idrossiapatite è infatti il fosfato più stabile sotto varie condizioni di $\mathrm{PH}$, eccetto quando questo diventa 
sufficientemente acido, ed in tal caso risulta stabile la brushite (POSSNER et al., 1984). In effetti i suoli argillosi, di per sé leggermente acidi, mescolati a guano, e quindi ancora più acidi, presentano generalmente brushite (CANCIAN, 1985).

L'idrossiapatite è stata rinvenuta anche nella Caverna Pocala (91 VG), ed in questo caso la sua origine può essere legata al disfacimento di un ricco deposito di ossa di fauna pleistocenica (Ursus Speleaus). Infine è stata rinvenuta anche in alcuni fanghi nerastri presenti sulle pareti della Caverna Generale Ricordi (1064 VG) preso Jamiano, in associazione a calcite e minerali argillosi.

Le soluzioni fosfatiche possono reagire anche con i minerali delle argille, formando la taranakite $-\left(\mathrm{K}, \mathrm{NH}_{4}\right) \mathrm{Al}_{3}\left(\mathrm{PO}_{4}\right)(\mathrm{OH})^{*} 9 \mathrm{H}_{2} \mathrm{O}-\mathrm{e} \mathrm{la}$ francoanellite $-\mathrm{H}_{6}(\mathrm{~K}, \mathrm{Na})_{3}(\mathrm{Al}, \mathrm{Fe})_{5}\left(\mathrm{PO}_{4}\right)_{8}{ }^{*} 13 \mathrm{H}_{2} \mathrm{O}$. Quest'ultimo minerale è piuttosto raro, essendo stato descritto fino ad oggi solo tre volte: nel Carso è presente nella Grotta Due Piani (4253 VG) (CHIORBOLI, 1984; CANCIAN, 1985).

\section{GLI OSSIDI ED IDROSSIDI DI FERRO E ALLUMINIO}

Sulle pareti e sulle volte delle grotte si notano spesso delle incrostazioni dure, di color ocra o marroncino, composte essenzialmente da ossidi ed idrossidi di ferro e secondariamente da quarzo ed argille; si tratta cioè di incrostazioni "limonitiche". Generalmente il minerale più abbondante è la goethite; sono stati riscontrati anche magnetite, maghemite ed ematite.

Anche nel suolo delle caverne sono stati riscontrati gli stessi minerali, però assieme a idrossidi di alluminio, quali la gibbsite, il diasporo e la bohemite.

\section{I CARBONATI}

Nelle cavità carsiche la calcite risulta il carbonato più frequente; molto rari risultano invece gli altri tipi di carbonati. Attualmente è in fase di studio uno straterello molliccio, di colore grigio, individuato nel suolo della Caverna Pocala (91 VG), in cui è stata rinvenuta un'associazione di calciokutnahorite, kutnahorite, aragonite, idrossiapatite e quarzo. La calcio-kutnahorite $\left[\mathrm{Ca} 0,{ }_{74}(\mathrm{Mn}, \mathrm{Mg})_{0,26} \mathrm{CO}_{3}\right]$ e la kutnahorite $\left[\mathrm{CaMn}\left(\mathrm{CO}_{3}\right)_{2}\right]$ sono due carbonati di $\mathrm{Ca}$ e $\mathrm{Mn}$ (gruppo della dolomite), finora rinvenuti in Italia nei "Limi di Terranova" nel Valdarno Superiore (BINI e MENCHETTI, 1985), in associazione ad aragonite e formatisi presumibilmente in se- 
guito a fenomeni pedogenetici legati principalmente a variazioni del livello basale dell'acqua.

Per quel che concerne la loro presenza nella caverna Pocala, si è ipotizzato che l'idrossiapatite, precipitata in seguito al disfacimento del deposito di ossa presente in questa caverna, reagendo con la calcite abbia causato una perdita di calcio in quest'ultima, compensata dall'entrata di manganese, presente nelle soluzioni circolanti al suolo. Questo fenomeno di sostituzione Ca-Mn ha portato alla formazione di kutnahoriti più o meno ricche di $\mathrm{Ca}$. Inoltre, dato che in queste soluzioni è presente anche dello stronzio (in seguito al già citato disfacimento delle ossa), il carbonato di calcio può precipitare anche sottoforma di aragonite, e ciò può spiegare la sua abbondanza entro questa Caverna. L'aragonite infatti oltre che nello straterello appena descritto, compare anche in masserelle pulverulente di colore biancastro, in associazione a calcite.

\section{IL GESSO}

Il gesso $\left(\mathrm{CaSO}_{4}{ }^{\star} \mathrm{H}_{2} \mathrm{O}\right)$ è un solfato idrato di calcio molto diffuso, ma praticamente sconosciuto nelle grotte del Carso. Al momento la sua presenza è stata accertata nella caverna Pocala, dove compare sotto forma di masserelle tenere di colore biancastro. Per quel che riguarda la sua genesi si possono formulare due ipotesi, e precisamente una riguarda la possibilità di avere nelle vicinanze dei solfati, la cui alterazione porti alla formazione di soluzioni da cui precipita il gesso, l'altra invoca fenomeni di tipo biologico, come decomposizione in ambiente anaerobico di sostanza organica, che portino alla formazione di $\mathrm{H}_{2} \mathrm{~S}$. Nel caso della caverna Pocala, la seconda ipotesi sembra essere la più consistente, dato che nel Carso non sono presenti solfati o solfuri, che per alterazione diano luogo a soluzioni da cui poi possa precipitare il gesso. 


\section{BIBLIOGRAFIA}

ANDREOLOTTI S. 1966: I depositi di riempimento nelle cavità del Carso triestino. Atti e Mem. Comm. Grotte Boegan, vol. V, Trieste.

ANDREOLOTTI S. 1970: Osservazione e descrizione di alcuni depositi di riempimento alluvionali in cavità e paleocavità del Carso triestino e istriano. Atti e Mem. Comm. Grotte Boegan, vol. IX, Trieste.

BINI B. AND MENCHETTI S. 1985: Kutnahorite from Levane Upper Valdarno (Italy). Per. di Miner., 54, 61-66.

CANCIAN G. 1981: Studio sui depositi alluvionali trovati nelle paleocavità e nella superficie del Carso goriziano. Le Grotte d'Italia, s. 4, vol. IX, Castellana Grotte.

CANCIAN G. 1985: I fosfati della Grotta ad Est di San Martino del Carso (Gorizia): brushite, idrossiapatite, francoanellite, taranakite. Atti III Conv. Triv. di Spel. (Vicenza 1984).

CANCIAN G. 1987: Ricerche mineralogiche sui fosfati delle grotte del Carso goriziano e triestino. Atti VII Conv. Reg. di Spel. del Friuli-Venezia Giulia, Gorizia.

CANCIAN G., CHIORBOLI S. AND LENZI G. 1986: Studio mineralogico preliminare delle "terre rosse" del Carso goriziano, monfalconese e triestino. Ist. di Min., Univ. di Ferrara, S.A.T.E.

Cancian G., Coltorti M. AND PRInCIVAlle F. 1990: Mineralogical study on hydroxylapatite encrustations in caves of the Gorizia Karst (Italy). Boll. Soc. Adr. Sc., LXXI, 21-28

CHIORBOLI S. 1984: Dati mineralogici preliminari sulla francoanellite $\mathrm{H}_{6} \mathrm{~K}_{3} \mathrm{Al}\left(\mathrm{PO}_{4}\right)_{8} \cdot 13 \mathrm{H}_{2} \mathrm{O}$ della Grotta Due Piani, San Martino del Carso (Gorizia). Ist. di Min. Univ. Ferrara, ed. Bortolazzi-Stei, S. Giovanni Lup. (Verona).

D’Ambrosi C. 1963: In merito all'assenza di depositi ghiaiosi sulle superfici carsiche della Venezia Giulia. Atti Mus. Civ. St. Nat. di Trieste, v. 24, n. 1.

FORTI F. 1973: Considerazione sui depositi di riempimento nelle cavità del Carso Triestino. Atti e Memorie Comm. Grotte Boegan, v. 13, Trieste.

Hill C. AND ForTI P. 1986: Cave minerals of the world. Nat. Speleol. Society, Cave avenue, Untsville, Alabama, USA.

POSSNER S.A., BLUMENTHAL C.N. AND BETTS F. 1984; Chemistry and structure of precipitated hidroxyl-apatites. In Nriagu J.O., Moore P.B.: Phospate Minerals, Springer Verlag, Berlin. 